\title{
Clinical Effectiveness of Percutaneous Full- endoscopy Spine Surgery Combined With Holmium Laser in the Treatment of Lumbar Disc Herniation
}

\section{Song Guo}

Shanghai General Hospital

Kai Zhu

Qingdao No. 8 People's Hospital

Fu Qiang (D2293259778@qq.com )

Shanghai General Hospital

Meiju Yan

Shanghai General Hospital

Donghua Hang

Shanghai General Hospital

\section{Research Article}

Keywords: Lumbar disc degeneration, Oxidative stress, Lower back pain, Holmium laser, Full-endoscopic spine surgery

Posted Date: December 10th, 2021

DOI: https://doi.org/10.21203/rs.3.rs-1141871/v1

License: (c) (i) This work is licensed under a Creative Commons Attribution 4.0 International License. Read Full License 


\section{Abstract}

Background Oxidative stress has been considered a critical mediator in the pathogenesis of lumbar disc degeneration, which can lead to the severe lower back pain. Inhibition of the excessive oxidative stress has become a therapeutic target for controlling discogenic lower back pain. Recently, more studies have shown that holmium laser can effectively suppress oxidative stress. This work aims to study the clinical outcomes of percutaneous full-endoscopy spine surgery combined with holmium laser for treating symptomatic lumbar disc degeneration (IDD).

Methods We designed and conducted a retrospective study on the patients with lumbar disc herniation who had been treated by percutaneous full-endoscopy spine surgery at Shanghai General Hospital from June 2018 to March 2020. The patients were divided into group A (holmium laser treatment group) and group $B$ (Elliquence radiofrequency group) according to the medical record. Operation time and hospitalization time of both groups were recorded and compared. Additionally, VAS scores and ODI scores were used to evaluate the lower back pain, lower extremity radiation pain and quality of life before and 3 days, 6 months, and 12 months after surgery.

Results Forty-five patients were included in group A while the other 55 patients were included in group B. Operation time of group A was significantly shorter than that of group B $(40.60 \pm 5.59$ minutes vs. $50.80 \pm 3.19$ minutes). VAS scores of lower back pain and lower extremity radiation pain at postoperative 3 days, 6 months, and 12 months were significantly lower than those before surgery in both groups. The postoperative lower back pain VAS scores of group A were significantly lower than those of group $B$, while the postoperative lower extremity radiation pain VAS scores had no significant difference between both groups. The ODI scores of both groups at postoperative 3 days, 6 months, and 12 months were significantly lower than those before surgery.

Conclusions These findings confirmed that application of holmium laser can significantly improve the clinical outcomes in percutaneous full-endoscopy spine surgery.

\section{Introduction}

Due to the aging population and change of living habits in the current society, the incidence of lumbar disc degeneration (IDD) increases annually. IDD causes severe lower back and leg pain, affecting the quality of life of patients and creates a large economic burden to patients and society $[1,2]$. Many factors, including genetic predisposition, smoking, infection, abnormal biomechanical loading and molecular mechanism, contribute to disc degeneration. Of which, the activation of oxidative stress was concluded to be a critical mediator in the pathogenesis of degenerative disc conditions and potential therapeutic target.

In recent years, holmium laser has been shown to inhibit oxidative stress and may play a key role in delaying IDD. Additionally, percutaneous full-endoscopic lumbar discectomy has been shown to achieve positive therapeutic effects on IDD by directly removing the herniated nucleus pulposus tissue and 
decompressing the nerve roots. This surgery prevents the need for vertebral fusion, which also prevents adjacent segment degeneration. As a result, percutaneous full-endoscopic lumbar discectomy has been used widely in the treatment of IDD [3-5]. Although endoscopic surgery has the advantages of less trauma, quick recovery, good curative effect and low cost, it still has some shortcomings such as low surgical efficiency, difficulty of hemostasis during surgery, and a long learning curve $[6,7]$. Elliquence radiofrequency has been adopted broadly as an intraoperative hemostatic and cutting tool clinically, which acts on the tissue to be cut through an emitter with a specific shape. The water molecules in the tissue cells oscillate rapidly under the action of radio waves, resulting in cutting [8]. Currently, the thermal injury around the cutting edges caused by the bipolar radiofrequency is $15 \mu \mathrm{m}$. In percutaneous fullendoscopic spine surgery, the surgery is concentrated primarily around the nerve root, so any form of thermal injury may lead to nerve root injury, thereby inducing catastrophic consequences. Hence, there is an urgent need to develop new endoscopic hemostasis and ablation tools for percutaneous fullendoscopic spine surgery to meet the requirements of efficient surgery, energy concentration and low thermal injury that reduces the risk of damage and improve surgical outcomes. Holmium laser can be used as the endoscopic hemostasis and ablation tool due to the decreased thermal injury, increased efficiency and positive effect on inhibiting oxidative stress in percutaneous full-endoscopic spine surgery. This retrospective clinical study was designed and implemented to investigate the efficacy of percutaneous full-endoscopic spine surgery combined with holmium laser in the treatment of IDD.

\section{Materials And Methods}

\section{General data}

Patients with lumbar disc degeneration who underwent percutaneous full-endoscopic spine surgery at the Shanghai General Hospital between June 2018 and March 2020 were analyzed retrospectively. The inclusion criteria involved: (1) patients with symptoms of low back pain complicated by radiating pain to the lower limbs; (2) those who had ineffective conservative treatment for three months, including physiotherapy, manual therapy and non-morphine therapy; (3) those with nerve root compression at a consistent location confirmed by CT or magnetic resonance imaging (MRI); (4) those whose images showed single segmental lumbar disc herniation; (5) those with L4-5 or L5-S1 lesions; (6) those who underwent unilateral percutaneous full-endoscopic lumbar discectomy; and (7) those without previous history of spinal surgery. The exclusion criteria were (1) patients with a previous history of tumor or spinal infection; (2) those with severe coagulation disorders, having a history of drug abuse, or taking anticoagulants orally; (3) those with contraindications for MRI (with an implantation history of cardiovascular or cerebrovascular stents, cardiac pacemaker or biological stimulator); (4) those who were pregnant or breast-feeding; (5) those who received interventional intervertebral disc therapy in the past three months, including radiofrequency treatment, laser ablation, protease injection or ozone injection; (6) those with a severe infection or high fever; or (7) those whose images showed severe spinal canal stenosis, lumbar spondylolisthesis, or other spinal deformities. This study was approved by the Ethics Committee of Shanghai General hospital (IACUC number: 2018AWS0015), and all subjects signed the 
informed consent. Additionally, this study has been performed in accordance with the Declaration of Helsinki since it involves human participants.

\section{Instruments and equipment}

Percutaneous full-endoscopic spine surgery and Elliquence radiofrequency systems were purchased from SPINEDOS (Munich, Germany). The holmium laser therapy system and laser sheath were sourced from Trimedyne (Irvine, USA), and the orthopedic C-arm fluoroscope was bought from Siemens (Munich, Germany).

\section{Surgical methods}

The patient lay prone on the all-carbon Allen spinal operation bed, and the lesion segment to be treated was determined by C-arm fluoroscopy. If the lesion segment was L4-5, the L4-5 intervertebral foramen was routinely punctured, and a working channel was established, followed by foraminoplasty. Later, the full-spinal endoscope was inserted, and ablation, electrocoagulation, hemostasis, removal of protruding nucleus pulposus tissue and loosening of the nerve root were performed using the holmium laser sheath or Elliquence radiofrequency. If the lesion segment was L5-S1, the posterior interlaminar approach and Carm fluoroscopy were adopted to determine the lesion segment, followed by establishment of a working channel and placement of a full-spinal endoscope. Thereafter, the holmium laser sheath or Elliquence radiofrequency was used for ablation, electrocoagulation, hemostasis, removal of protruding nucleus pulposus tissue and loosening of the nerve root. The holmium laser sheath was used for tissue ablation and hemostasis in group A (holmium laser power range: 20-60 W, frequency range: $15-20 \mathrm{~Hz}$, energy range: 1-1.5 J). Elliquence radiofrequency was employed for cutting and hemostasis in group $B$. The choice of cutting and hemostasis instruments was based on different time periods, and no other selection criteria were used in the study.

\section{Post-operative treatment}

The day after surgery, the patients were instructed to carry out rehabilitation exercises but were prescribed mostly bed rest for four weeks after surgery. They were instructed to use a brace when out of bed. Meanwhile, the patients were advised to avoid strenuous activities, bending and bearing weight in the short term after surgery but to exercise and strengthen their lower back muscles to prevent recurrence of symptoms.

\section{Major observation indices}

For both groups: (1) Intraoperative nerve decompression, ablation of fat, soft tissue, ligamentum flavum, intervertebral disc and bone surface, and hemostatic effects were recorded. (2) The operation time and hospital stay were recorded. (3) The VAS scores for lower back pain and lower limb radiating pain before treatment were recorded and at 3 days, 6 months and 12 months after treatment. (4) The Oswestry Disability Index (ODI) score was recorded before treatment and at 3 days, 6 months, and 12 months after treatment. With the VAS score, the pain degree was expressed by numbers from 0 to 10,0 for no pain and 
10 for the most pain. The patients picked one number from 0-10 to represent their pain degree. ODI was used to evaluate the improvement of patients' viability. The ODI scale included 10 items involving pain intensity, self-care ability of daily life, lifting, walking, sitting, standing, sleeping, sex life, social life and travelling, with 6 options for each question scoring 0-5 points. Evaluation was based on a total score of 50 points: ODI score $=$ actual score $/ 50 \times 100 \%$. The higher the score, the more severe the dysfunction.

\section{Statistical analysis}

SPSS 18.0 software was utilized for statistical analysis. The measurement data were expressed as mean \pm standard deviation. One-way analysis of variance was employed to compare all parameters between groups, LSD tests were performed for pairwise comparisons within groups. Chi-square tests were used to compare rates between groups. $\mathrm{P}<0.05$ was considered statistically significant.

\section{Results}

\section{Analysis of general data}

According to the above inclusion and exclusion criteria, 100 patients were enrolled and divided into group A (Fig. 1) ( $n=45$, undergoing percutaneous full-endoscopic spine surgery combined with holmium laser treatment group, Fig. 2 and Fig. 3), or group $B(n=55$, undergoing percutaneous full-endoscopic spine surgery combined with Elliquence radiofrequency treatment). In group A, there were 22 males and 23 females, aged 18-67 years old, with an average age of $46.40 \pm 11.63$ years old. There were 25 patients with L4-5 lesions and 20 patients with L5-S1 lesions. In group B, there were 27 males and 28 females, aged 1969 years old, with a mean age of $48.44 \pm 11.67$ years old. There were 28 cases with L4-5 lesions and 27 cases with L5-S1 lesions. Statistical analysis revealed no significant differences in the baseline data for gender, age, or lesion segment between the two groups.

\section{Follow-up results}

All subjects were followed for $12-24$ months, with a mean of 19.6 months.

\section{Ablation and hemostatic effect}

The ablation of fat, soft tissue, ligamentum flavum, intervertebral disc and bone surface, and the hemostatic effect, in group A were significantly better than those in group B (Fig. 2). Group A had a better outcome of the intervertebral discoplasty and markedly reduced herniated intervertebral discs after surgery (Fig. 3).

\section{Operation time and hospital stay}

The operation time in group A was significantly shorter than that in group B $(40.60 \pm 5.59$ min. vs. $50.80 \pm 3.19$ min., respectively $\mathrm{P}<0.05$ ) (Fig. 4). However, there was no significant difference in the hospital stay between groups ( $3.89 \pm 1.25$ days $v s .4 .34 \pm 1.43$ days, respectively). 


\section{VAS scores}

The VAS scores for lower back pain and lower limb radiating pain at 3 days, 6 months and 12 months after surgery dropped significantly compared with those before surgery in both groups $(P<0.05)$ (Fig. 5). An intergroup comparison showed that the VAS score for postoperative lower back pain in group $A$ was significantly lower than that in group $B(P<0.05)$ (Fig. 6). Nevertheless, there was no significant difference in the VAS score for postoperative lower limb radiating pain between the two groups.

\section{ODI scores}

The ODI scores 3 days, 6 months, and 12 months after surgery were significantly reduced compared with those before surgery in both groups $(P<0.05)$ (Fig. 7). However, there was no significant difference in the postoperative ODI score between the two groups.

\section{Discussion}

Laser energy can be greatly absorbed by water in human tissues, which makes the water molecules boil and the intracellular and extracellular fluid evaporate rapidly, resulting in vaporization, ablation, and hemostasis. Due to advantages such as high energy and low penetration, lasers have been applied widely in for surgery in dermatology, urology, and other medical subspecialties. Netsch et al used a holmium laser to remove benign prostatic hyperplasia and found that the holmium laser safely and effectively ablated hyperplastic prostate tissue peri-operatively and obtained good clinical results [9]. Zhu et al compared the efficacy and safety of the holmium laser and trans-urethral resection in primary nonmuscular invasive bladder tumors and concluded that holmium laser treatment could markedly improve surgical efficiency, reduce peri-operative complications and improve safety in bladder tumor surgeries [10].

The application of lasers in spinal surgery is still in its early phase. In 1987, Choy et al used Nd:YAG laser ablation of herniated nucleus pulposus tissue to complete lumbar intervertebral disc decompression. However, the decompression was not carried out under direct vision, so there was a great risk of nerve root injury [11]. With the development of percutaneous endoscopic technology, the percutaneous laser transmission system is combined with spinal endoscopy, the intervertebral disc tissue and nerve structure can be directly observed by the intervertebral foramen or interlaminar approach, and laser vaporization and ablation can be used to remove the nucleus pulposus tissue and decompress nerve roots more accurately, safely, and thoroughly. So far, multiple types of lasers have been used clinically, including the neodymium laser (Nd:YAG), holmium laser (Ho:YAG) and semiconductor laser [12, 13]. Among them, the wavelength of the holmium laser is $2.1 \mu \mathrm{m}$, the depth of tissue penetration is only $\leq 0.5 \mu \mathrm{m}$, and the beam is easily absorbed by water. Compared with other types of laser, the holmium laser creates less thermal damage to surrounding tissues such as the dural sac and nerve roots so it is more widely adopted in clinically. In the study of Ruetten et al [14], the holmium laser was combined with spinal epidural endoscopic surgery. When the holmium laser energy was controlled at $0.8 \mathrm{~J}$ (frequency $8 \mathrm{~Hz}$ ), epidural scar tissues could be cut effectively and safely, improving the symptoms of chronic low back pain. 
Daehyun et al studied the risk of dural damage caused by the holmium laser in epidural endoscopy and found in cadaver experiments that when the holmium laser energy was set at $5 \mathrm{~J}$, the holmium laser irradiation perpendicular to the dura induced more obvious dural damage compared with irradiation parallel to the dura. In addition, the degree of dural injury was more severe with more prolonged irradiation time. Therefore, they suggested that attention should be paid to the risk of dural injury when using the holmium laser in spinal epidural endoscopy [15].

Holmium laser technology has been widely applied in epidural endoscopy, but the application of the holmium laser in full-endoscopic spine surgery is still in its infancy. Holmium laser surgery and fullendoscopic spine surgery need "water" as the working medium, so the efficient combination of holmium laser technology and full-endoscopic spine surgery can provide advantages of both techniques, improving surgical efficiency and safety. Yeung et al [16] reported that endoscopic resection of the nucleus pulposus and decompression with the holmium laser was performed through the postero-lateral "Kambin triangle" approach to the intervertebral disc. The results of at least one-year follow-up after surgery showed that the rate of excellent and good clinical results was $83.6 \%$. As a reliable tool for endoscopic minimally invasive surgery, the holmium laser can effectively complete rapid vaporization, ablation, and hemostasis of different structures such as the ligamentum flavum, nucleus pulposus, annulus fibrosus and bone wounds, and shorten the operation time for exposing the surgical target area and removing the intervertebral disc tissues. Meanwhile, during precise ablation, it can reduce the risk of nerve injury because it avoids most tissue injuries. Moreover, the holmium laser lateral opening is beneficial for dealing with the "blind area" in the long and narrow endoscopic channel that includes the horizontal dorsal ligamentum flavum of the intervertebral disc. Knight et al [17] reported that 48 patients with lumbar lateral recess stenosis underwent enlarged intervertebral foramenoplasty with the holmium laser endoscopically. This quickly removes hyperplastic osteophytes, protruding nucleus pulposus and epidural scar tissue, thus improving surgical efficiency.

One of the major technical difficulties in full-endoscopic spine surgery is intraoperative hemostasis. Hemostasis of the bone surface after arthroplasty is particularly difficult, and the visual field disturbance caused by surgical bleeding increases the operation time and risk. In traditional Elliquence radiofrequency surgery, it is necessary to place the radiofrequency tip on the tissue and convert its electrical energy into thermal energy so that the local tissue temperature rises to $60^{\circ} \mathrm{C}$, causing protein denaturation and achieving hemostasis. As the conversion speed of electric energy to heat energy is slow, and the local tissue temperature rises slowly, the hemostatic efficiency of traditional Elliquence radiofrequency is low. In contrast, the holmium laser has high energy, the conversion of light energy to thermal energy is faster, and the energy can be quickly absorbed by the tissue to reach the hemostatic temperature, which makes the local tissue coagulate. In this study, it was found that the holmium laser effectively stopped bleeding not only of the soft tissue, but also of the bone surface after articular process resection. Effective intraoperative hemostasis can further improve safety and shorten surgical time. In this study, the operation time in group A (receiving the holmium laser treatment) was significantly shorter than that in group B (receiving traditional Elliquence radiofrequency treatment). 
Clinically, lumbar disc herniation often is accompanied by discogenic low back pain caused by disc degeneration in addition to radiating pain to the lower limbs. However, $15-25 \%$ of patients still have discogenic lower back pain after nucleus pulposus removal [18]. Full-endoscopic spine surgery can remove the herniated nucleus pulposus tissue, relieve nerve root compression, and effectively improve the symptoms of pain radiating to the lower limbs. However, whether full-endoscopic spine surgery can effectively relieve discogenic lower back pain is still controversial. In the study of Gibson et al [19], 70 patients with lumbar disc herniation underwent trans-foraminal percutaneous endoscopic lumbar discectomy (PELD), and their VAS scores for lower back pain and leg pain decreased from $5.7 \pm 2.7$ points and $6.2 \pm 2.8$ points before surgery to $2.5 \pm 2.5$ points and $1.9 \pm 2.6$ points two years after surgery, respectively. Ruetten et al [20] reported that among the 232 patients with lumbar disc herniation after PELD, the improvement rate of radiating pain in the lower limbs was $96 \%$, but lower back pain was not improved significantly compared with pre-operative pain. Ten patients needed open surgery due to persistent low back pain after the initial surgery.

To further clarify the improvement of lower back pain and lower limb radiating pain after full-spinal endoscopy combined with holmium laser surgery, the VAS scores for lower back pain and lower limb radiating pain were recorded, respectively. The preoperative pain symptoms were similar in the two groups. Postoperative lower back pain was relieved by full-spinal endoscopy in both groups, indicating that "flowing water" as a surgical medium in full-endoscopic spine surgery can remove chronic paincausing substances accumulated in the "herniated intervertebral disc" effectively [18]. In addition, whether the holmium laser or Elliquence radiofrequency was used in the intervertebral disc, thermal effects are produced, inhibiting inflammatory factors that cause pain and achieving local physiotherapy to relieve the symptoms of low back pain in both groups [21]. However, in this study, the VAS scores for lower back pain in the holmium laser treatment group were significantly lower than those in the Elliquence radiofrequency treatment group at all time points after surgery. We hypothesized that the tissue damage in the intervertebral disc nucleus pulposus and annulus fibrosus caused by the holmium laser was significantly smaller than that using Elliquence radiofrequency, thus reducing the secretion of inflammatory factors such as prostaglandins and interleukins that stimulate pain nerve fibers [22]. In addition, the concentration of holmium laser energy can accurately inactivate granulation tissue and nociceptive nerve fibers in the annulus fibrosus, and further reduce pain in the intervertebral disc. Last but not least, lumbar nucleus pulposus (NP) resides in a hypoxic microenvironment, which is essential for maintaining normal cellular metabolism and protein synthesis. Once the blood vessel grows into the NP and disrupts the hypoxic microenvironment, the subsequent oxidative stress induces NP degeneration to cause lower back pain. The expression of oxidative stress markers, including pentosidine and advanced glycation end products (AGEs), is increased in the degenerative human NP [23]. Excessive reactive oxygen species (ROS) was concluded to be a critical mediator in the pathogenesis of degenerative disc conditions and potential therapeutic target. Antioxidant NAC significantly abrogated the catabolic effect of excessive ROS in vitro and in vivo. Therefore, oxidative stress occurred in lumbar NP played a critical role in accelerating degeneration to cause discogenic lower back pain. Inhibition of the excessive oxidative stress has become a therapeutic target for controlling discogenic lower back pain. Recently, 
more studies showed laser was widely for the cosmetic surgery due to its effective inhibition on oxidative stress. Weng et al. indicated laser treatment increased the level of enzymatic antioxidant including uperoxide dismutase (SOD) and glutathione peroxidase (GSH-Px) to elucidate the mechanism of laser nonablative rejuvenation in the established primary skin fibroblasts model [24]. Additionally, low-level laser therapy demonstrated favorable effects in modulating the oxidative stress by decreased ROS and increased activity of antioxidant enzymes such as superoxide dismutase (SOD) and glutathione peroxidase (GPx) to improve regenerative capacity of dystrophic muscle cells in the experimental model of Duchenne muscular dystrophy (DMD) [25]. Therefore, holmium laser improves the symptoms of discogenic lower back pain by potential reducing oxidative stress in the NP. There was no significant difference in the VAS score for postoperative lower limb radiating pain or the ODI score between the two groups, indicating that either the holmium laser or Elliquence radiofrequency can achieve effective nerve decompression in a full-endoscopic spine surgery and relieve the symptoms of lower limb radiating pain and improve the quality of life of the patients.

Although the holmium laser was applied initially in full-endoscopic spine surgery and achieved good clinical results, there are still some shortcomings in the application of the holmium laser in fullendoscopic spine surgery. Firstly, the current holmium laser generator has the disadvantages of large volume, noise, difficulty of fixing the laser sheath during surgery, and it causes pollution easily. Secondly, the lack of minimally invasive instruments matched with laser surgery causes difficulties during surgery and prolongs the operative time. Thirdly, holmium laser parameters need to be further studied to ensure the optimal effect of tissue ablation and hemostasis. Lastly, the mechanism of improved postoperative lower back pain and inhibition effects of oxidative stress of the holmium laser needs to be further investigated. At present, there is an urgent need to develop a new laser generator and related minimally invasive instruments to further improve operation efficiency and safety, facilitate the population for which full-spinal endoscopy combined with holmium laser surgery, and improve the therapeutic effect in patients with lumbar disc herniation, thus relieving the burden of patients and society.

\section{Conclusions}

Application of holmium laser can significantly improve the surgical efficiency, reduce operation time and relieve lower back pain symptom in percutaneous full-endoscopy spine surgery.

\section{Declarations}

\section{Ethical Approval:}

This study was performed in line with the principles of the Declaration of Helsinki. Approval was granted by the Ethics Committee of Shanghai General Hospital (May 5, 2018).

\section{Consent to participate:}

Informed consent was obtained from all individual participants included in the study. 


\section{Consent to publish:}

The authors affirm that human research participants provided informed consent for publication of the images.

\section{Authors Contributions:}

Guo Song: acquisition of data, Drafting the manuscript

Zhu Kai: analysis and/or interpretation of data:

Fu Qiang: Conception and design of study

Yan Meijun: Drafting the manuscript

Hang Donghua: Validation and Methodology

\section{Funding:}

This study was funded by the National Natural Science Foundation of China (81971154).

\section{Competing Interests:}

The authors declare that there are no conflict of interests.

\section{Availability of data and materials:}

All data generated or analyzed during this study are included in this published article and available from the corresponding author upon reasonable request.

\section{Acknowledgements:}

The authors would like to express their gratitude to EditSprings (https://www.editsprings.com/) for the expert linguistic services provided.

\section{References}

1. Walker BF. The prevalence of LBP. A systematic review of the literature from 1966 to 1998. J Spinal Disord. 2000;13:205-17.

2. Choi YS. Pathophysiology of degenerative disc disease. Asian Spine J. 2009;3: 39-44.

3. Chen Z, Zhang L, Dong J et al. Percutaneous Transforaminal Endoscopic Discectomy Versus Microendoscopic Discectomy for Lumbar Disc Herniation: Two-Year Results of a Randomized Controlled Trial. Spine. 2020;45(8):493-503.

4. Hyeun SK, Jong DY, Chang IJ. Predictive Scoring and Risk Factors of Early Recurrence After Percutaneous Endoscopic Lumbar Discectomy. Biomed Res Int 2019;doi: 10.1155/2019/6492675. 
eCollection 2019.

5. Hao L, Li S, Liu J et al. Recurrent Disc Herniation Following Percutaneous Endoscopic Lumbar Discectomy Preferentially Occurs When Modic Changes Are Present. J Orthop Surg Res. 2020;doi: 10.1186/s13018-020-01695-6.

6. Liang JQ, Chen C, Zhao H. Revision Surgery after Percutaneous Endoscopic Transforaminal Discectomy Compared with Primary Open Surgery for Symptomatic Lumbar Degenerative Disease. Orthop Surg. 2019;11(4):620-7.

7. Shi R, Wang F, Hong $X$ et al. Comparison of percutaneous endoscopic lumbar discectomy versus microendoscopic discectomy for the treatment of lumbar disc herniation: a meta-analysis. Int Orthop. 2019;43(4):923-37.

8. Giovanni G, Fabio T, Alessandro L. Prevention of Lumbar Reherniation by the Intraoperative Use of a Radiofrequency Bipolar Device: A Case-Control Study. J Craniovertebr Junction Spine. 2019;10(2):949.

9. Netsch C, Becker B, Tiburtius $\mathrm{C}$ et al. A prospective, randomized trial comparing thulium vapoenucleation with holmium laser enucleation of the prostate for the treatment of symptomatic benign prostatic obstruction: perioperative safety and efficacy. World J Urol. 2017; 35:1913-21.

10. Zhu YF, Jiang XZ, Zhang JP, et al. Safety and Efficacy of Holmium Laser Resection for Primary Nonmuscle-Invasive Bladder Cancer Versus Transurethral Electroresection: Single-Center Experience. Urology. 2008;72(3):608-12.

11. Choy DS, Case RB, Ascher P et al. Percutaneous Laser Nucleolysis of Lumbar Disks [J]. N Engl J Med. 1987; 317(12):771-2.

12. Moon BJ, Lee HY, Kim KN, et al. Experimental Evaluation of Percutaneous Lumbar Laser Disc Decompression Using a 1414 nm Nd:YAG Laser. Pain Physician. 2015;18(6):E1091-9.

13. Kim BJ, Ahn J, Cho H, et al. Early Individualised Manipulative Rehabilitation Following Lumbar Open Laser Microdiscectomy Improves Early Post-Operative Functional Disability: A Randomized, Controlled Pilot Study. J Back Musculoskelet Rehabil. 2016;29(1):23-9.

14. Ruetten S, Meyer O, Godolias $G$ et al. Application of holmium:YAG laser in epiduroscopy: extended practicabilities in the treatment of chronic back pain syndrome. J Clin Laser Med Surg. 2002;20(4):203-6.

15. Daehyun J, Lee D. The Extent of Tissue Damage in the Epidural Space by Ho/YAG Laser During Epiduroscopic Laser Neural Decompression. Pain Physician. 2015;18:E209-E14.

16. Yeung AT, Tsou PM. Posterolateral endoscopic excision for lumbar disc hemiation: surgical technique, outcome, and complications in 307 consecutive cases $₫$. Spine (Phila Pa 1976). 2002;27(7):722-31.

17. Knight MT, Vajda A, Jakab GV et a1. Endoscopic laser foralllinoplasty on the lumbar spine-eady elperience. Minim Invasive Neurosurg. 1998;41(1):5-9.

18. Liu KC, Yang SK, Ou BR. et al. Using Percutaneous Endoscopic Outside-In Technique to Treat Selected Patients with Refractory Discogenic Low Back Pain. Pain Physician. 2019;22(2):187-98. 
19. Gibson JNA, Subramanian AS, Scott CEH. A randomised contonlled trial of transforaminal endoscopic discectomy vs microdiscectomy. Eur Spine J. 2017;26(3):847-56.

20. Ruetten S, Komp M, Merk H, et al. Endoscopic Lumbar Decompression Full-endoscopic Interlaminar and Transforaminal Lumbar Discectomy Versus Conventional Microsurgical Technique: A Prospective, Randomized, Controlled Study. Spine (Phila Pa 1976).2008;33(9):931-9.

21. Manabe H, Yamashita K, Tezuka F. et al. Thermal Annuloplasty Using Percutaneous Endoscopic Discectomy for Elite Athletes with Discogenic Low Back Pain. Neurol Med Chir (Tokyo).2019;59(2):48-53.

22. Ryu JJ, Yoo S, Kim KY, et al. Laser modulation of heat and capsaicin receptor TRPV1 leads to thermal antinociception. J Dent Res. 2010;89(12):1455-60.

23. Sivan S, Tsitron E, Wachtel E, et al. Age-related accumulation of pentosidine in aggrecan and collagen from normal and degenerate human intervertebral discs. Biochem J. 2006;399:29-35.

24. Weng Y, Dang Y, Ye XY et al. Investigation of irradiation by different nonablative lasers on primary cultured skin fibroblasts. Clinical \& Experimental Dermatology. 2011;36(6):655-60.

25. Macedo AB, Moraes LHR, Mizobuti DS et al. Low-Level Laser Therapy (LLLT) in Dystrophin-Deficient Muscle Cells: Effects on Regeneration Capacity, Inflammation Response and Oxidative Stress. PLoS ONE. 2015; Doi: 10.1371/journal.pone.0128567.

\section{Figures}

Cohort of patients with lumbar disc degeneration

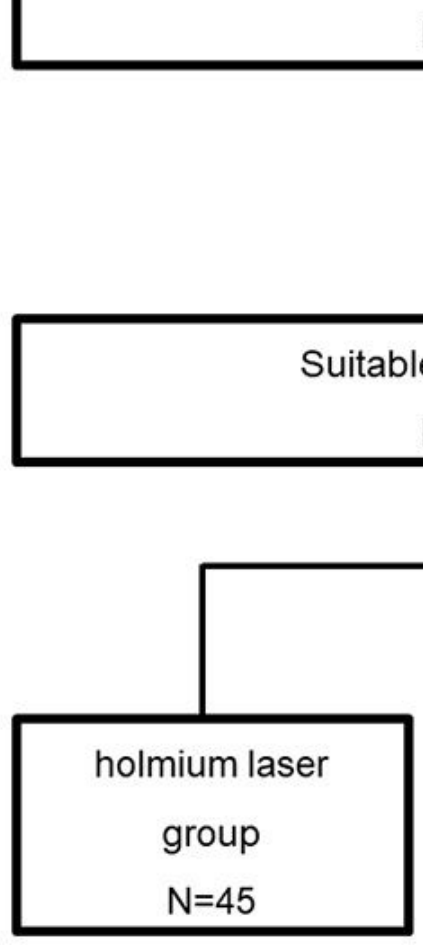

$\mathrm{N}=132$
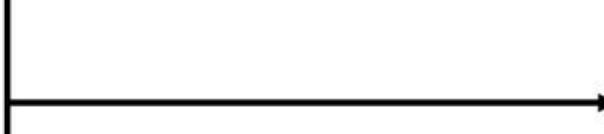

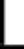

inclusion

$\mathrm{N}=100$

Divided
Excluded

history of tumor or spinal infection:2

severe coagulation disorders: 2

contraindications for MRI :5

pregnant or breast-feeding; 2

severe infection or high fever:2

severe spinal canal stenosis: 10

lumbar spondylolisthesis: 5

spinal deformities: 4

choice of cutting and hemostasis

instruments based on different time periods 


\section{Figure 1}

Flow chart diagram of patient selection.

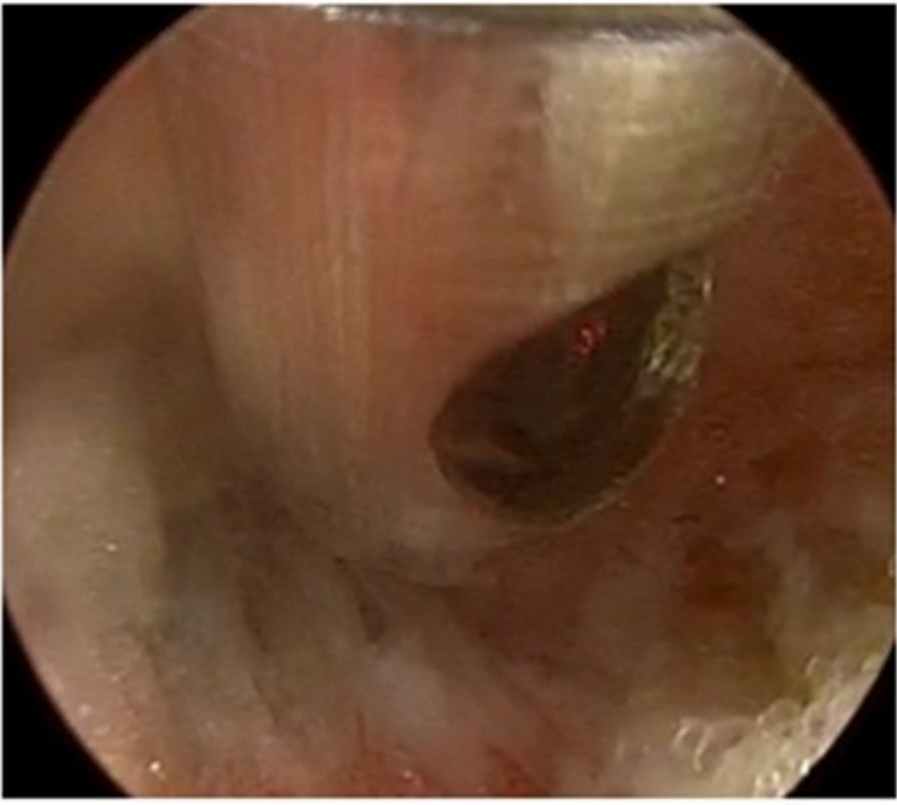

Fat ablation

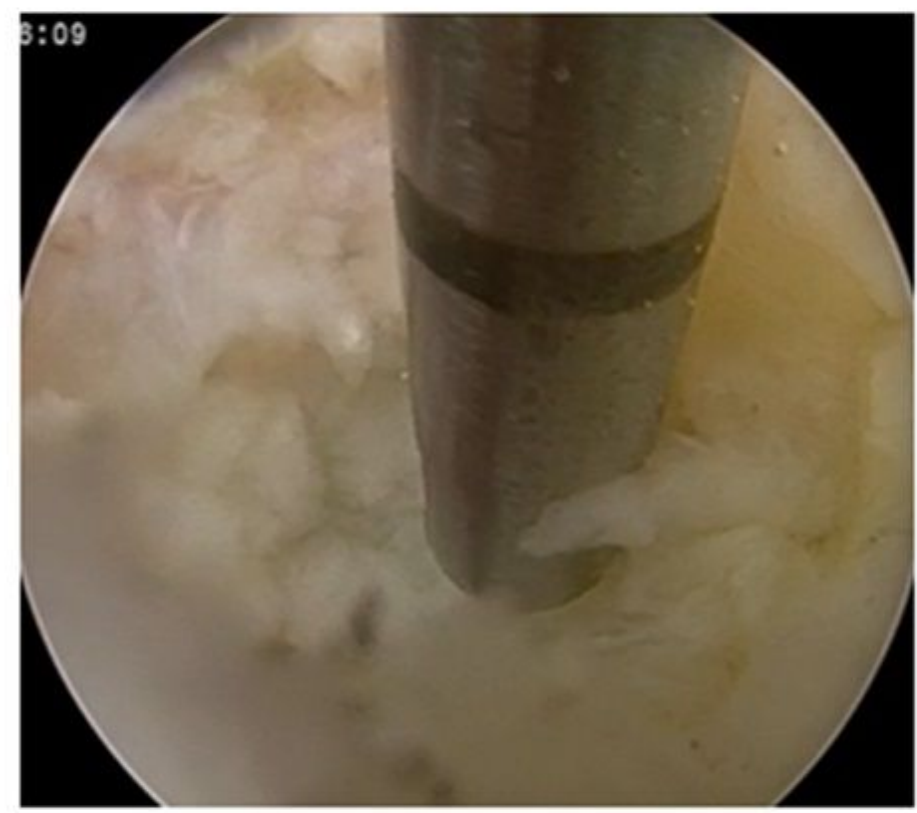

intervertebral disc ablation

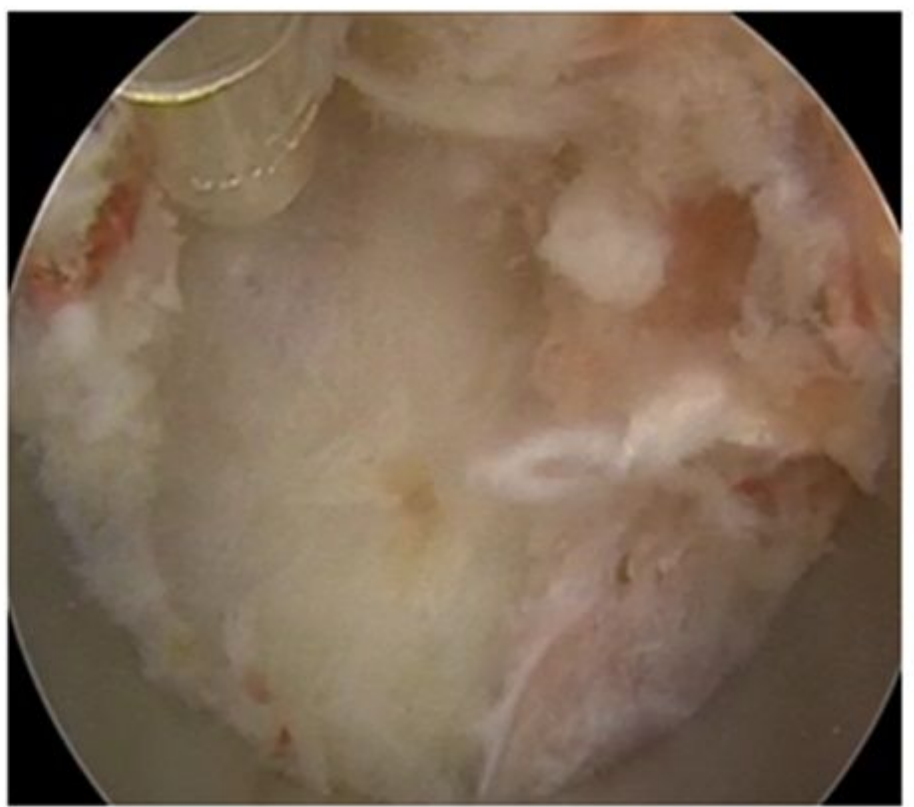

\section{Soft tissue ablation}

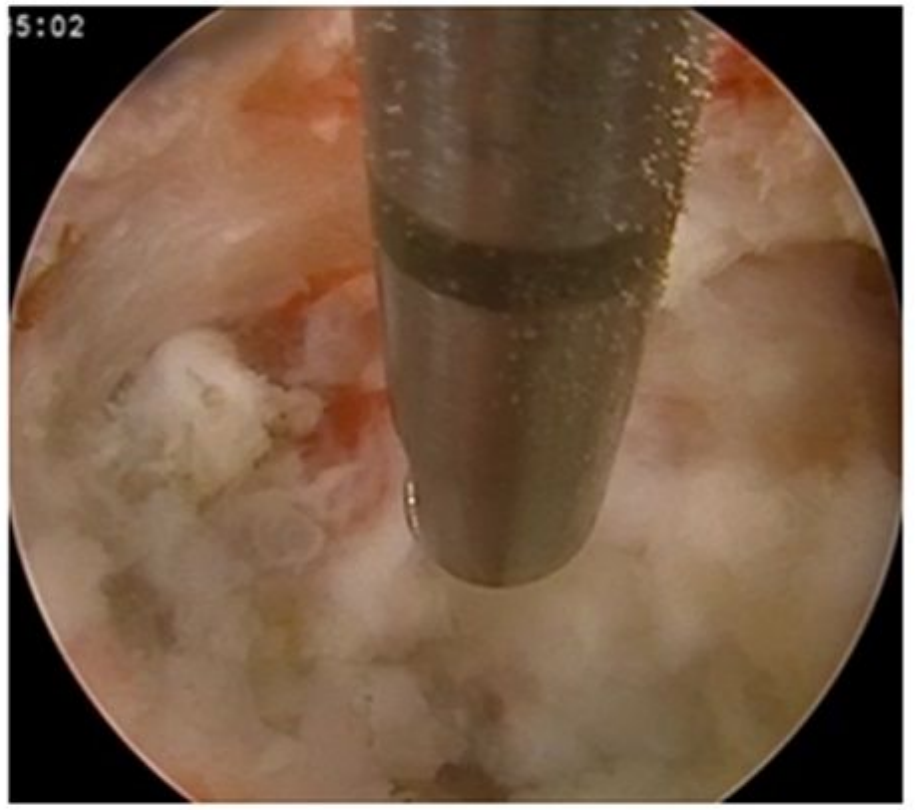

\section{bone surface hemostasis}

Figure 2

Holmium laser is used for the ablation and hemostasis of fat, soft tissue, intervertebral disc and bone surface during full-endoscopy spine surgery. It can be seen that this holmium laser is side-shot during operation. 


\section{Figure 3}

Imaging data of patients with typical lumbar disc herniation (L4-5) treated with holmium laser. A-C: preoperative CT showed huge disc herniation compressing the left nerve root; D-F: preoperative MRI L4-5 giant intervertebral disc herniation compressing the left nerve root; G-I: MRI after full-endoscopy spine surgery combined with holmium laser showed that the herniated nucleus pulposus disappeared.

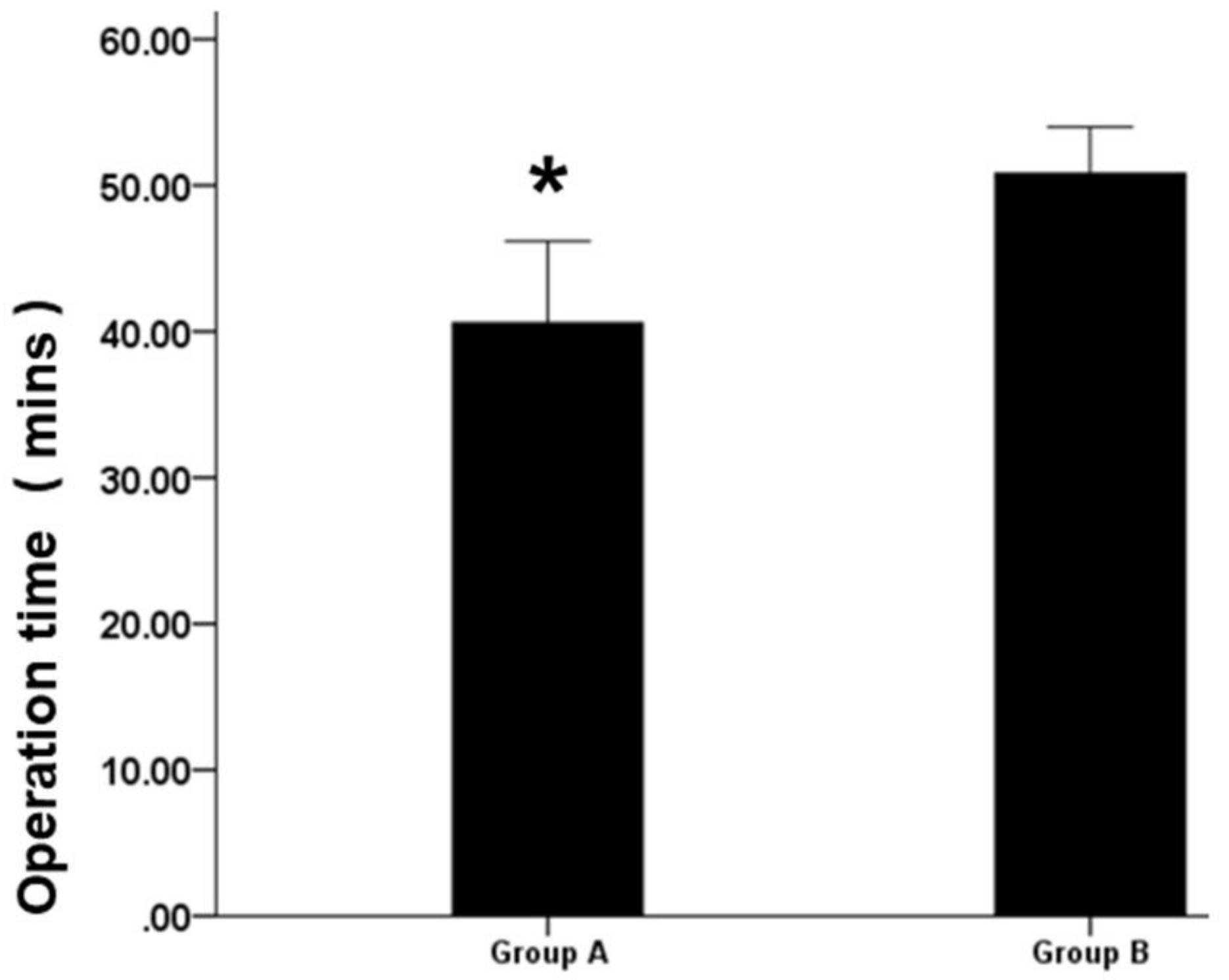

Figure 4

The difference in operation time between the two groups: the operation time of group A was significantly shorter than that of group $B(P<0.05)$. 

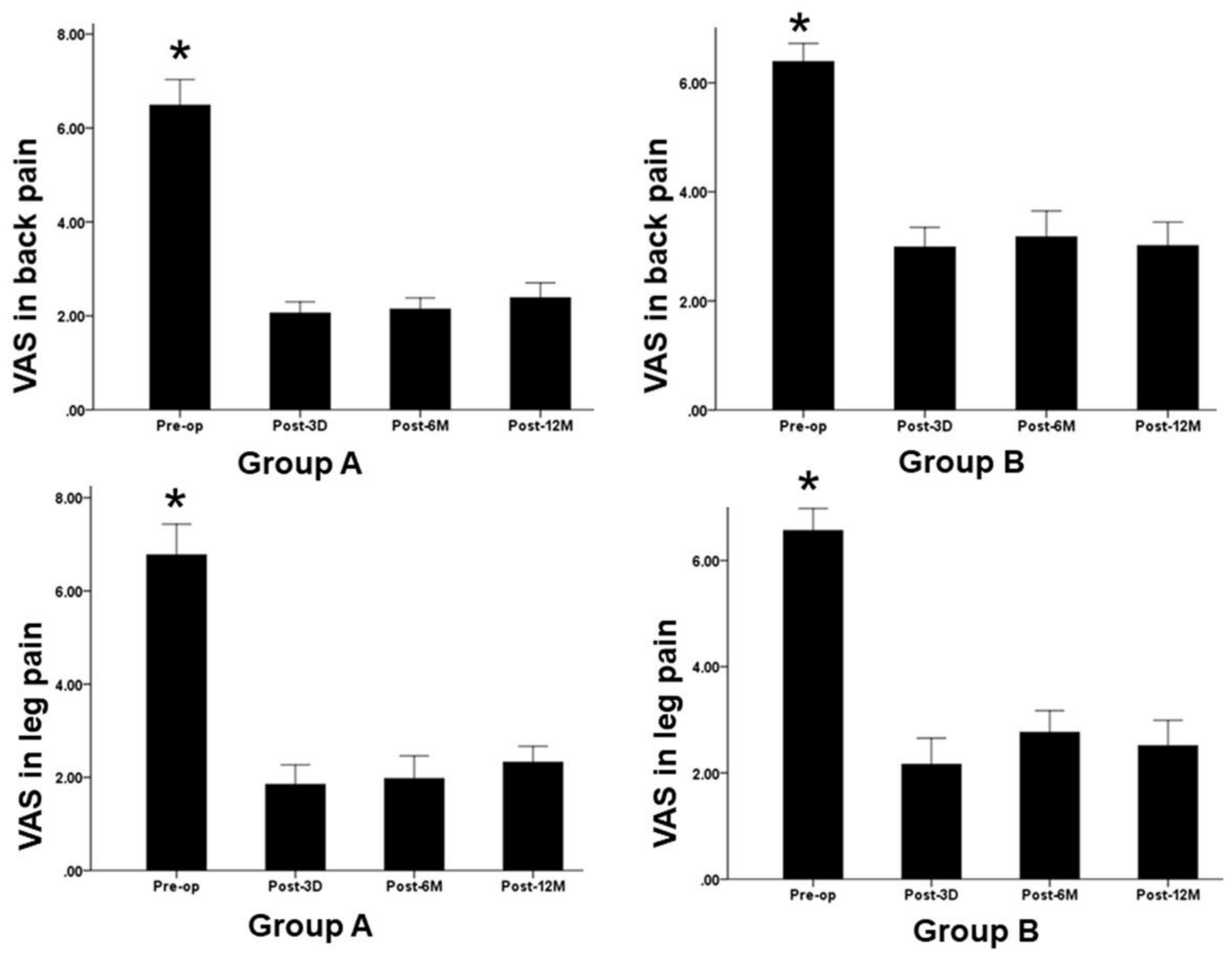

Figure 5

The preoperative and postoperative lower back pain VAS scores and lower limb radiating pain scores of the two groups: the VAS scores of lower back pain and lower limb radiating pain at the 3rd-day, 6th-month and 12th-month postoperative were significantly lower than those before surgery $(P<0.05)$. 

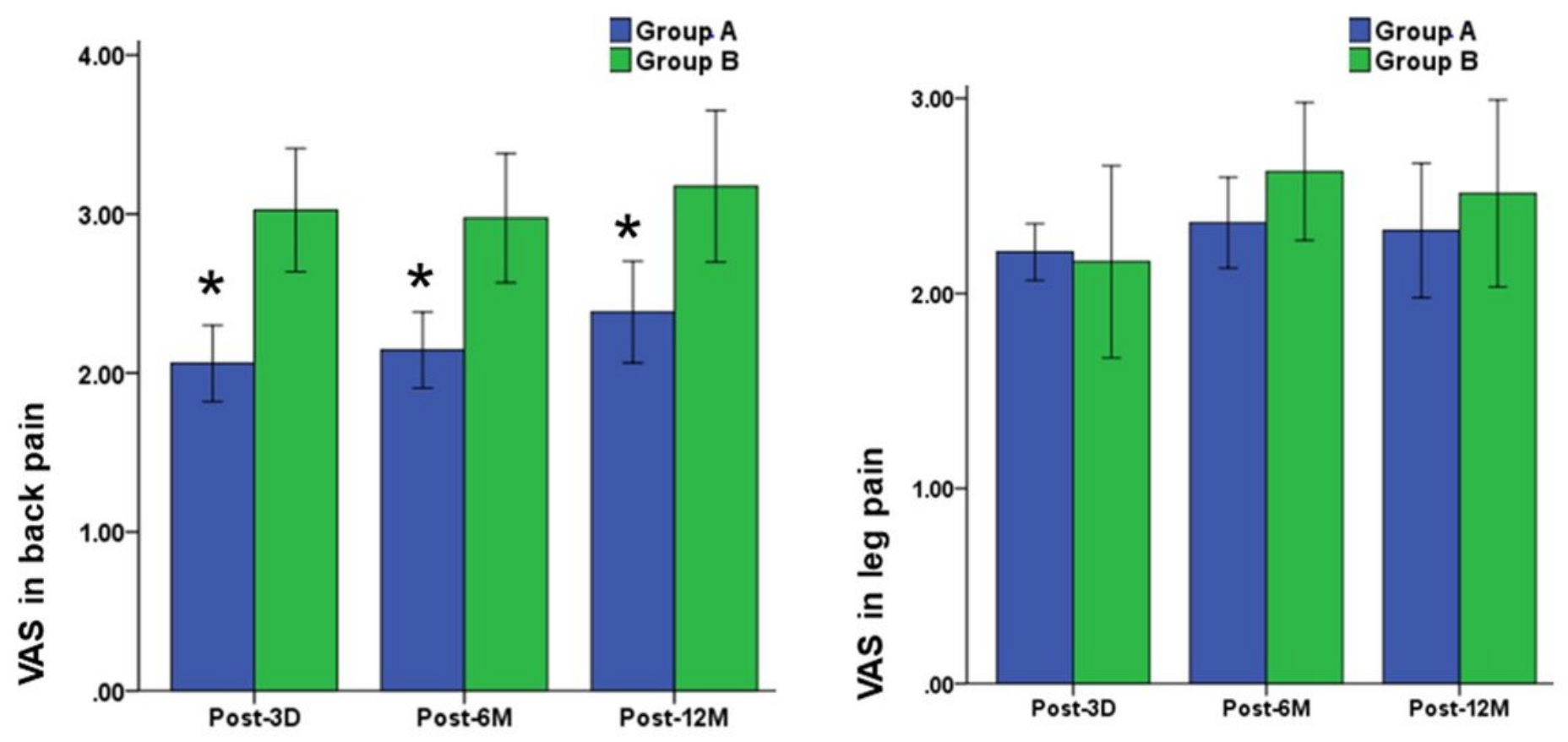

Figure 6

Comparison of postoperative lower back pain and lower limb radiating pain between the two groups: showed that the VAS score of postoperative lower back pain in group A was significantly lower than that of group $B(P<0.05)$; there was no difference in the VAS score of postoperative lower limb radiating pain between the two groups $(P>0.05)$.
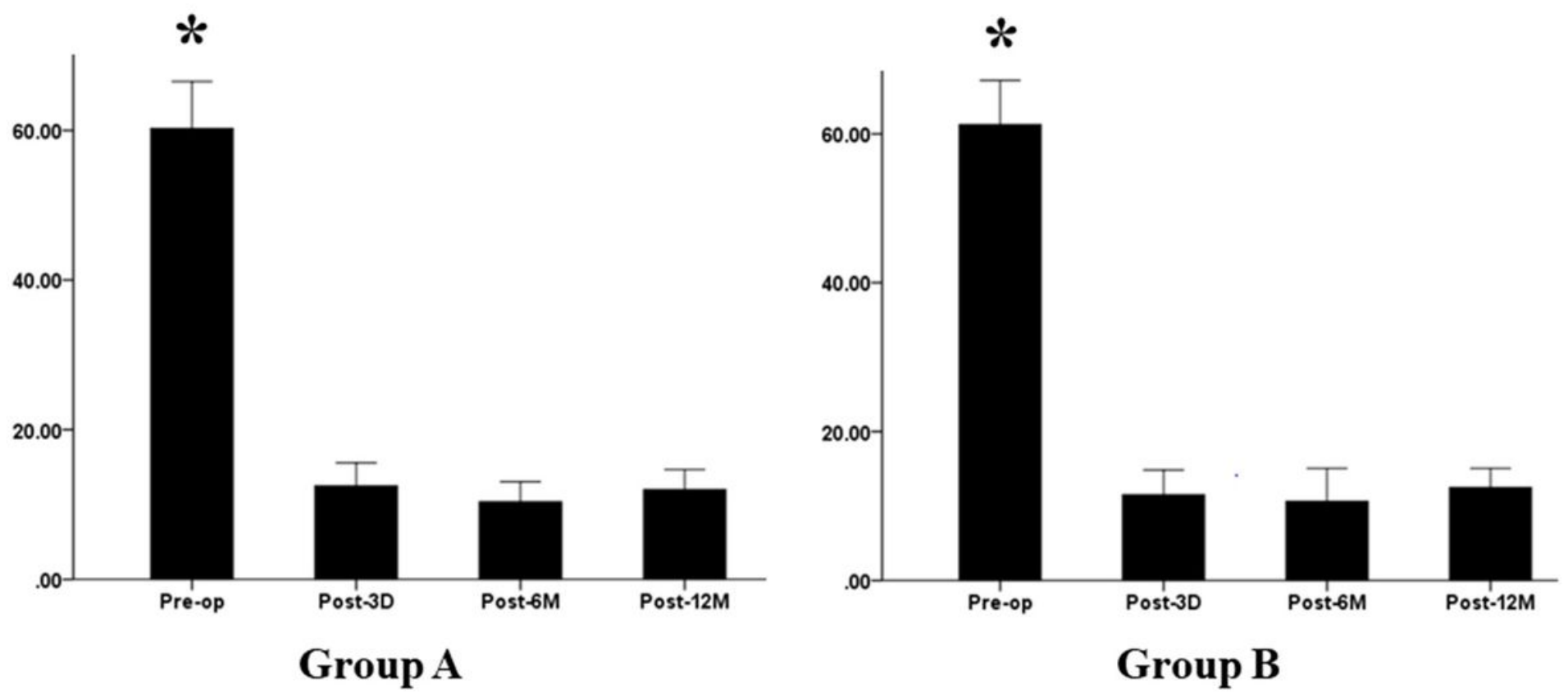

Figure 7 
The preoperative and postoperative quality of life (ODI) scores of the two groups: the ODI scores of the two groups at the 3rd-day, 6th-month and 12th-month postoperative follow-up were significantly lower than those before surgery $(P<0.05)$. 